\title{
Non-LTE modelling of prominence fine structures using hydrogen Lyman-line profiles
}

\author{
P. Schwartz ${ }^{1,3,4}$, S. Gunár ${ }^{2, \dagger}$, and W. Curdt ${ }^{3}$ \\ 1 Astronomical Institute of Slovak Academy of Sciences, 05960 Tatranská Lomnica, Slovak Republic \\ e-mail: pschwartz@astro.sk \\ 2 School of Mathematics and Statistics, University of St. Andrews, North Haugh, St. Andrews, Fife, KY16 9SS, UK \\ 3 Max-Planck-Institut für Sonnensystemforschung, Justus-von-Liebig-Weg 3, 37077 Göttingen, Germany \\ 4 Astronomical Institute, Academy of Sciences of the Czech Republic, 25165 Ondřejov, Czech Republic
}

Received 10 October 2014 / Accepted 10 March 2015

\begin{abstract}
Aims. We perform a detailed statistical analysis of the spectral Lyman-line observations of the quiescent prominence observed on May 18, 2005.

Methods. We used a profile-to-profile comparison of the synthetic Lyman spectra obtained by 2D single-thread prominence finestructure model as a starting point for a full statistical analysis of the observed Lyman spectra. We employed 2D multi-thread finestructure models with random positions and line-of-sight velocities of each thread to obtain a statistically significant set of synthetic Lyman-line profiles. We used for the first time multi-thread models composed of non-identical threads and viewed at line-of-sight angles different from perpendicular to the magnetic field.

Results. We investigated the plasma properties of the prominence observed with the SoHO/SUMER spectrograph on May 18, 2005 by comparing the histograms of three statistical parameters characterizing the properties of the synthetic and observed line profiles. In this way, the integrated intensity, Lyman decrement ratio, and the ratio of intensity at the central reversal to the average intensity of peaks provided insight into the column mass and the central temperature of the prominence fine structures.
\end{abstract}

Key words. Sun: filaments, prominences - line: profiles - radiative transfer - methods: statistical

\section{Introduction}

Spectral observations of the quiescent solar prominences in hydrogen Lyman lines and continuum represent a unique tool for probing of their structure and physical properties. A substantial amount of prominence spectral data in Lyman lines was provided by the Solar Ultraviolet Measurements of Emitted Radiation (SUMER) UV-spectrograph (Wilhelm et al. 1995) onboard the Solar and Heliospheric Observatory (SoHO) satellite. Prominence observations made with instruments onboard SoHO were reviewed by Patsourakos \& Vial (2002); see also a review of the prominence fine structures by Heinzel (2007).

Interpreting the prominence observations in the Lyman lines requires sophisticated models of the prominence plasma in the magnetic field and complex non-LTE radiative transfer computations. Such models were first developed by Poland \& Anzer (1971) and Heasley \& Mihalas (1976). Later, Gouttebroze et al. (1993) used a sophisticated hydrogen atom with more than 20 levels plus continuum and a large grid of 1D isothermal isobaric slab models. This was followed by Anzer \& Heinzel (1998, 1999), who developed 1D magnetohydrostatic equilibrium models of the Kippenhahn-Schlüter (K-S) type. These were generalized into 2D by Heinzel \& Anzer (2001), whose prominence fine-structure models take the form of $2 \mathrm{D}$ vertically infinite plasma threads in magnetohydrostatic equilibrium,

$\dagger$ S. Gunár is on a long-term leave from Astronomical Institute of the Academy of Sciences of the Czech Republic. suspended in magnetic dips. The temperature structure of these prominence fine-structure threads is described semi-empirically and includes the prominence-corona transition region (PCTR). The substantial influence of the PCTR on the Lyman-line intensities was demonstrated by Anzer \& Heinzel (1999). Twodimensional vertically infinite prominence fine-structure models, but with the cylindrical cross-section, were also developed by Gouttebroze (2006).

The importance of the multi-thread prominence finestructure models was shown by Gunár et al. (2007), who used a trial-and-error method to reproduce the observed Lyman spectrum. Later, Gunár et al. (2008) added random line-of-sight (LOS) macroscopic velocities to each thread of the multi-thread model, thus showing that even relatively low LOS velocities (of about $10 \mathrm{~km} \mathrm{~s}^{-1}$ ) have a significant effect on the asymmetries of the Lyman line profiles. Multi-thread models with randomly distributed LOS velocities of individual threads were shown by Gunár et al. (2010) to produce synthetic Lyman spectra that agree well with observations on the profile-to-profile as well as on statistical bases. Moreover, Berlicki et al. (2011) and Gunár et al. (2012) showed that the synthetic $\mathrm{H} \alpha$ spectra produced by multi-thread prominence finestructure models also agree with the observations. Furthermore, Gunár et al. (2011a) and Gunár et al. (2011b) demonstrated that the temperature and electron density structures of these models lead to the synthetic differential emission-measure curves that agree well with the observations. Recently, Gunár et al. (2014) 

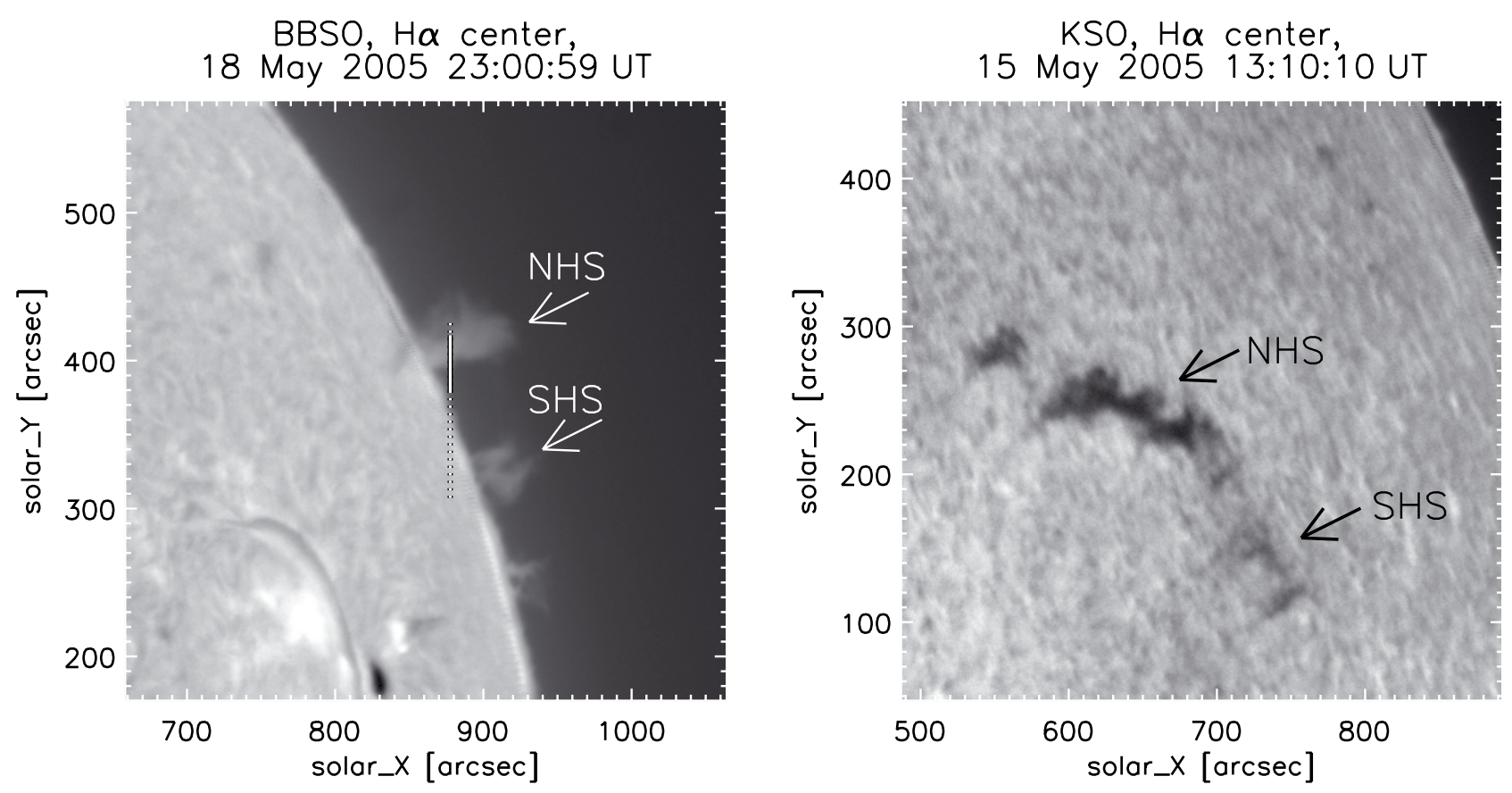

Fig. 1. Left panel: part of the full-disk $\mathrm{H} \alpha$ line-centre filtergram obtained by BBSO/NST on 18 May 2005. Vertical bar marks the position of the SoHO/SUMER slit. Spectra were obtained only from the part of the slit that is marked by the solid line because the SUMER detector was partially damaged. Right panel: prominence seen as a filament in part of the full-disk observations in the $\mathrm{H} \alpha$ line centre made at the Kanzelhöhe Solar Observatory three days earlier. The two clearly visible $\mathrm{H} \alpha$ features marked by arrows and denoted as NHS and SHS are the northern and southern $\mathrm{H} \alpha$ prominence structures.

combined the multi-thread prominence fine-structure models with the $3 \mathrm{D}$ prominence magnetic field modelling to analyse a prominence observed on 22 June 2010.

Current reviews of the prominence physics can be found in Labrosse et al. (2010) and Mackay et al. (2010) and also in the review of the prominence fine-structure modelling by Gunár (2014).

In Sect. 2 we describe the observations in detail. Section 3 provides details of the prominence fine-structure modelling. In Sect. 4 we present our results, Sect. 5 contains the discussion, and Sect. 6 provides the conclusions.

\section{Observations}

A quiescent prominence was observed on the NW limb of the Sun by the SUMER spectrograph onboard the SoHO satellite. The observations were made during the fifteenth MEDOC observing campaign between 18 May 2005 22:01:12 and 19 May 2005 01:37:19 UT with static slit position (sit-and-stare observations). The position of the SoHO/SUMER spectrograph slit is shown in the left panel of Fig. 1 on the $\mathrm{H} \alpha$ line-centre filtergram from National Solar Telescope (NST; Goode et al. 2003) at Big Bear Solar Observatory (BBSO). The co-alignment was achieved by correlating the distributions of BBSO/NST H $\alpha$ data and the integrated Ly $\alpha$ SoHO/SUMER intensities along the slit. Usable SUMER data were obtained from only 40 pixels on the northern part of the SUMER slit because other part of detector A was damaged. The usable part of the slit, marked by the full line in Fig. 1, crosses a large column-like prominence structure oriented radially, which contains cool $(T \leq 10000 \mathrm{~K})$ plasma where the $\mathrm{H} \alpha$ line is formed. Another similar smaller structure is visible to the south. In Fig. 1 we mark these two features as the northern and southern $\mathrm{H} \alpha$ prominence structures.
Table 1. Properties of the SOHO/SUMER observations.

\begin{tabular}{ccccc}
\hline \hline $\begin{array}{c}\text { Spectral } \\
\text { window }\end{array}$ & $\begin{array}{c}\text { Wavelength } \\
\text { range } \\
{[\AA]}\end{array}$ & $\begin{array}{c}\text { Slit dimensions } \\
(\text { width } \times \text { height }) \\
{[\operatorname{arcsec}]}\end{array}$ & $\begin{array}{c}\text { Exp. } \\
\text { time } \\
{[\mathrm{s}]}\end{array}$ & $\begin{array}{c}\text { No. } \\
\text { of obs. }\end{array}$ \\
\hline Ly $\alpha$ & $1175.0-1218.5$ & $0.28 \times 119.6$ & 15 & 18 \\
Ly $\beta$ & $987.3-1032.8$ & $0.99 \times 119.6$ & 98 & 36 \\
Ly $\gamma$, Ly $\delta$ & $932.0-977.7$ & $0.99 \times 119.6$ & 98 & 36 \\
Ly5 to & & & & 36 \\
Continum & $900.2-946.0$ & $0.28 \times 119.6$ & 98 & 36 \\
\hline
\end{tabular}

Notes. The table includes: spectral window, wavelength range, slit dimensions, exposure time, and number of observations.

In the right panel of Fig. 1 the prominence is seen as a filament in the $\mathrm{H} \alpha$ filtergram made two days earlier by the Patrol Telescope for Solar Observations located at the Kanzelhöhe Solar Observatory. The two $\mathrm{H} \alpha$ prominence structures are clearly visible as two individual filamentary objects marked by arrows.

Complete hydrogen Lyman series were observed by SUMER in four spectral windows: $1215.6 \AA, 1025.0 \AA, 972.0 \AA$, and $940 \AA$, each with a wavelength range of approximately $45 \AA$ and a cadence of 1-2 min. The basic properties of this set of observations are shown in Table 1 . The observed spectra were reduced and calibrated using standard SolarSoft procedures. The spectral lines were identified using the SUMER spectral atlas of Curdt et al. (2001).

We note that the spectral image on the detector is inclined with respect to the direction of dispersion as a result of the different orientation of the grating and the detector. This causes 


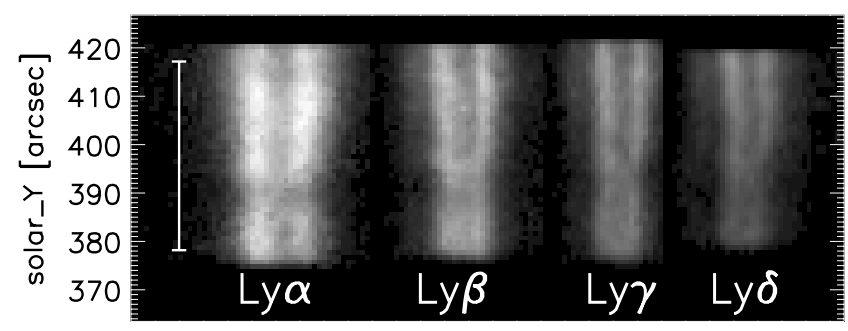

Fig. 2. Calibrated spectra of the $\operatorname{Ly} \alpha-\operatorname{Ly} \delta$ lines. The spectra were corrected for vertical shifts. The vertical white bar marks the usable part of the slit.

two spectral lines with different wavelengths to be shifted with respect to each other. Moreover, displacement of the slit image on the detector due to non-linearity of the grating focus mechanism causes additional cross-dispersion shifts (Schühle 2003). Vertical shifts of the $\operatorname{Ly} \beta$ and the higher Lyman lines with respect to the Ly $\alpha$ line were estimated by a comparison of the integrated intensity distributions and line profile shapes along the slit. Owing to the low signal-to-noise ratio in the spectra of the Ly 5 and higher Lyman lines, only profiles of the $\operatorname{Ly} \alpha-\operatorname{Ly} \delta$ lines were used in our analysis. An example of calibrated and corrected spectra of the four Lyman lines from one observation of the sit-and-stare observing sequence is shown in Fig. 2.

The Ly $\alpha$ line was placed on a so-called bare part of the detector $\mathrm{A}$ - outside the $\mathrm{KBr}$ coating and the attenuator. To protect the detector from further damage caused by high Ly $\alpha$ intensity, a lower detector voltage of $5390 \mathrm{~V}$ (standard is $5656 \mathrm{~V}$ ) was set during the Ly $\alpha$ observations. As was shown by Schwartz et al. (2012), calibration is only marginally affected (by less than 2\%) at such lower voltage. Other Lyman lines were observed with the standard voltage. A similar set of Lyman-line observations with lower voltage for the $\operatorname{Ly} \alpha$ line was also used by Gunár et al. (2010).

\section{Prominence fine-structure modelling}

Quiescent solar prominences are not homogeneous, but are composed of numerous fine structures, often quasi-vertical threads (see e.g. the review by Heinzel 2007). To analyse the Lyman spectra observations of these structures, we used the 2D prominence fine structure model of Heinzel \& Anzer (2001) in the multi-thread configuration including the random LOS velocities of individual threads developed by Gunár et al. (2008). In this section we briefly describe the single- and multi-thread models and show the results of the statistical comparison of the synthetic Lyman line profiles with observed data.

\subsection{Single-thread model}

The 2D prominence fine structure model of Heinzel \& Anzer (2001) approximates prominence quasi-vertical threads by a vertically infinite 2D structure embedded in a horizontal dipped magnetic field. The plasma in the thread is in the local magnetohydrostatic (MHS) equilibrium of Kippenhahn-Schlüter type. The thread is symmetrically irradiated at all four sides by diluted (height-dependent) radiation from the solar surface. The dimension across the magnetic field $-y$ coordinate - is fixed, but the dimension along the field $-x$ coordinate - is determined by the MHS equilibrium. The centre of the coordinate system is fixed in the centre of the thread. Instead of the geometrical $x$-scale, the column mass $m$-scale was used in the direction along the magnetic field. The total column mass $M(y)$ along a magnetic field line at a position $y$ is defined as (Heinzel \& Anzer 2001)

$$
M(y)= \begin{cases}M_{0}\left(1-\left|\frac{y}{\delta}\right|^{\gamma_{3}}\right) & \text { for }|y| \leq \delta \\ 0 & \text { for }|y|>\delta,\end{cases}
$$

where $M_{0}$ is the column mass at the centre of the thread (at $y=$ $0), \delta$ is half of the dimension of the thread across the magnetic field, and $\gamma_{3}$ is the exponent describing the column mass gradient across the magnetic field. We assumed here $\gamma_{3}=2$. The $2 \mathrm{D}$ temperature structure of the thread in $m-y$ scale is defined as (Heinzel \& Anzer 2001)

$$
\begin{aligned}
T(m, y)=T_{\mathrm{cen}}(y)+\left[T_{\mathrm{tr}}-T_{\mathrm{cen}}(y)\right] & \left\{1-4 \frac{m}{M(y)}\right. \\
& \left.\times\left[1-\frac{m}{M(y)}\right]\right\}^{\gamma_{1},}
\end{aligned}
$$

where $T_{\text {tr }}$ is the temperature at the thread boundary and $\gamma_{1}$ is the exponent describing the temperature gradient along the magnetic field. $T_{\text {cen }}(y)$ is the temperature at the dip centre at a position $y$ and is defined as (Heinzel \& Anzer 2001)

$T_{\text {cen }}(y)= \begin{cases}T_{\text {tr }}-\left(T_{\text {tr }}-T_{0}\right)\left(1-\left|\frac{y}{\delta}\right|^{\gamma_{2}}\right) & \text { for }|y| \leq \delta \\ T_{\text {tr }} & \text { for }|y|>\delta,\end{cases}$

where $T_{0}$ is the minimum temperature at the thread centre $(x=$ $0, y=0)$ and $\gamma_{2}$ is the exponent describing the temperature gradient across the magnetic field. This together with two additional input parameters of the model - the gas pressure $p_{0}$ at the thread boundary and the horizontal component of the magnetic field $B_{x}(0)$ in the dip centre - defines the distribution of the gas pressure $p(x, y)$ in the thread.

Thus, the $2 \mathrm{D}$ vertical-thread model of the thread has 11 input parameters: the column mass $M_{0}$, the dimension across the magnetic field $\Delta y=2 \delta$, the temperatures at the boundary $T_{\text {tr }}$ and at the centre $T_{0}$, the central horizontal magnetic field $B_{x}(0)$, the boundary gas pressure $p_{0}$, the exponents $\gamma_{1}, \gamma_{2}, \gamma_{3}$, the abundance of helium $A(\mathrm{He})$ with respect to hydrogen (here we used 0.1 , and the ratio of the microturbulence and sound velocities $\varepsilon$ (we adopted $\varepsilon=0.5$ ). We assumed the dimension of the threads in the direction across the magnetic field $2 \delta$ to be $1000 \mathrm{~km}$. Temperatures in the PCTR reach up to the coronal values, but because hydrogen spectral lines are formed at temperatures well below $100000 \mathrm{~K}$, we adopted this value for $T_{\mathrm{tr}}$. Examples of the temperature and gas-pressure distributions in the 2D thread are shown in Fig. 3.

We solved the 2D non-LTE radiative transfer by employing the Multilevel Accelerated Lambda Iterations (MALI; Auer \& Paletou 1994) in the short characteristics method (Kunasz \& Auer 1988). To solve the statistical equilibrium, we used a hydrogen atom with 12 levels plus continuum. Partial frequency redistribution (PRD) was assumed for the $\operatorname{Ly} \alpha$ and $\operatorname{Ly} \beta$ lines. For the higher Lyman lines the complete frequency redistribution was assumed.

\subsection{Multi-thread model}

As was shown by Gunár et al. (2007), the observed intensities in the peaks and near wings of the $\operatorname{Ly} \beta$ and higher Lyman lines are better reproduced by multi-thread prominence fine-structure models than by models with only one thread. At the same time, the intensities in the centre of these lines produced by singlethread and multi-thread models are the same. This can be explained by the fact that in the line centre, where optical thickness is very large, only radiation from the foremost fine-structure 

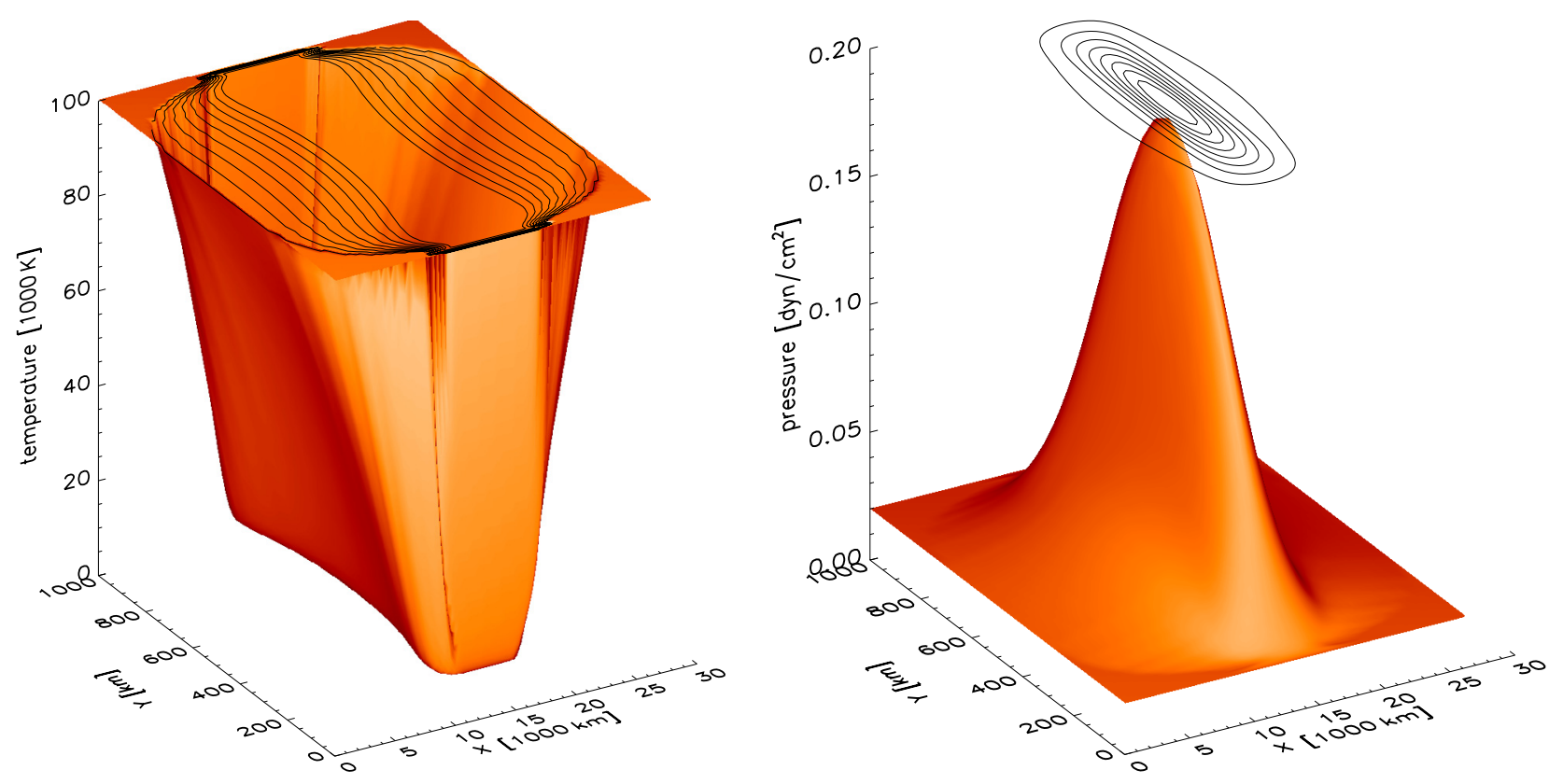

Fig. 3. Example of the temperature and gas-pressure distributions produced by the $2 \mathrm{D}$ single-thread model with the best agreement with observed spectra. The temperature step between contours is $10000 \mathrm{~K}$, that of the gas pressure is $0.02 \mathrm{dyn} / \mathrm{cm}^{2}$.

thread can be seen. In contrast, the optical thickness in peaks is much lower and thus also the radiation from the threads farther away from the observer contributes to the measured intensities. This effect is more pronounced for the higher members of the Lyman line series, but is negligible for the Ly $\alpha$ line because of its large optical thickness even in the near wings. The multi-thread model used in this work (Gunár et al. 2008) was constructed in such a way that the foremost thread (the one closest to the observer) was fixed in the centre of the computational domain. The other threads were shifted to the left or right along the magnetic field with a maximum displacement equal to the half of the $x$-dimension of the thread. The distance between the threads was fixed to $1000 \mathrm{Km}$. The mutual irradiation of threads was not taken into account. As was shown by Gunár et al. (2008), assigning various LOS velocities to individual threads of such a multi-thread model can explain the observed asymmetries in the Lyman-line profiles.

The total intensity $I_{\lambda}$ (total) along a given LOS emerging from the multi-thread model composed of $N$ threads with random LOS velocities (see scheme in Fig. 4) was calculated using the following recurrent formula (derived from Eq. (1) of Gunár et al. 2008) with $j$ increasing from 2 to $N$ :

$I_{\lambda}^{\mathrm{em}}(j)=I_{\lambda}^{\mathrm{em}}(j-1) \times \exp \left(-\tau_{\lambda}(j)\right)+I_{\lambda}(j)$,

where $I_{\lambda}^{\mathrm{em}}(1)=I_{\lambda}(1)$ and $I_{\lambda}($ total $)=I_{\lambda}^{\mathrm{em}}(N)$. The intensity emerging from the $j$ th thread of the multi-thread model is denoted as $I_{\lambda}^{\mathrm{em}}(j), I_{\lambda}(j)$ is the intensity radiated by the $j$ th thread itself, and $\tau_{\lambda}(j)$ is its optical thickness. The latter two quantities are defined in the observer's frame as

$$
\begin{aligned}
I_{\lambda}(j) & \equiv I(\lambda-\Delta \lambda(j)) \\
\tau(j) & \equiv \tau(\lambda-\Delta \lambda(j)) .
\end{aligned}
$$

The quantity $\Delta \lambda(j)$ is the Doppler shift for the $j$ th thread corresponding to its LOS velocity $\xi(j)$ :

$$
\Delta \lambda(j)=\lambda_{0} \frac{\xi(j)}{c}
$$

where $\lambda_{0}$ is the central wavelength of a given spectral line and $c$ the speed of light in vacuum. Positive values of $\xi(j)$ represent velocities oriented towards the observer. Here we used LOS velocities from the interval of $\pm 10 \mathrm{~km} \mathrm{~s}^{-1}$. Equation (4) can be adapted to obtain the total emerging intensities also along the LOS not perpendicular to the magnetic field. In this case, parameter $\mu$ (the cosine of the angle between the actual LOS and the direction perpendicular to the magnetic field) is introduced.

We obtained the distributions of the absorption and emission coefficient for individual threads with the 2D single-thread model described in the previous section. The $I_{\lambda}(j)$ intensities for the given value of $\mu$ were calculated by the quadrature form of Mihalas et al. (1978) for the formal solution of radiative transfer. More details can be found in Gunár et al. (2008).

\subsection{Statistical comparison of observed and synthetic profiles}

Previously, Gunár et al. (2010) used a 2D multi-thread model composed of identical threads for a statistical analysis of the promincence observed on May 25, 2005. Here we employed the multi-thread models with identical and, for the first time, also non-identical threads. For the non-identical threads we assumed higher values of the central minimum temperatures $T_{0}$ in threads at the edges of the multi-thread model. Moreover, we used for the first time the LOS angles different from the perpendicular to the magnetic field in the statistical analysis. We do not aim to determine the exact configuration of the multi-thread model with randomly generated shifts and LOS velocities of individual threads. To perform the statistical analysis, we instead estimated the input parameters of the single-thread model by comparing the synthetic and observed Ly $\alpha$ profiles. The Ly $\alpha$ line is suitable for this because of its large optical thickness, which means that its profiles are contributed mainly from the foremost thread of any multi-thread setup. The multi-thread model is then constructed from individual threads with input parameters determined in this way. The same method was used by Gunár et al. (2010). When synthetic Lyman line profiles for a large number of realizations of the multi-thread model are calculated, the 


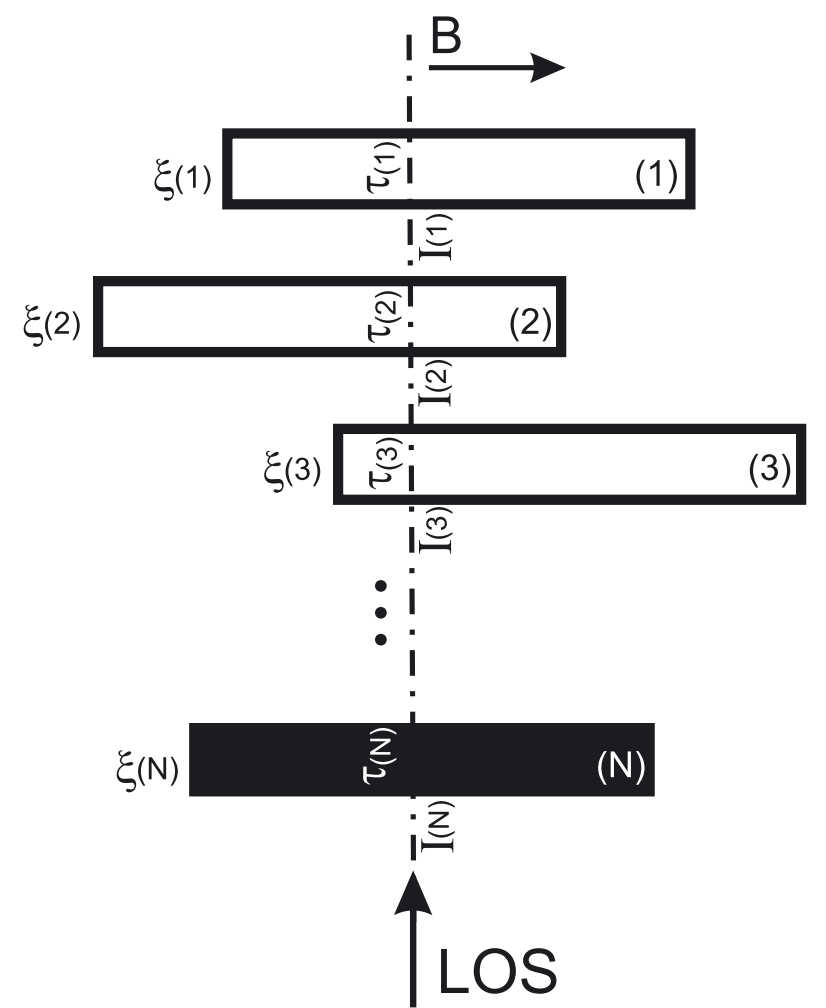

Fig. 4. Scheme of the prominence multi-thread model composed of $N$ threads and LOS perpendicular to the magnetic field. Quantities $\xi(j)$ represent the LOS velocities of individual threads.

characteristics of the synthetic profiles can be statistically compared with large sets of observed profiles. Multiple observations of prominences with a static slit (sit-and-stare mode) are suitable for such a study. We used three statistical characteristics of the Lyman-line profiles: integrated intensity, Lyman decrement ratio of the integrated intensity of a given line to the $\operatorname{Ly} \beta$ integrated intensity, and depth of the reversal - ratio of intensity at the central reversal to average intensity of peaks (the so-called centre-to-peak ratio). The centre-to-peak ratio for both the synthetic and observed profiles was determined as follows (see also Gunár et al. 2010): first, positions of the two peaks in a profile were found and the ratio was calculated by dividing the minimum intensity in wavelength interval between the peaks by the mean intensity of the peaks. To eliminate the influence of the noise on the estimation of the center-to-peak ratio, average intensities from three wavelength pixels at the reversal minimum and at each peak were used in the observed data. For profiles without reversal, the centre-to-peak ratios were not estimated, and these profiles were excluded from the statistical analysis of the reversal depth, but not from the analysis of the other two parameters. Gunár et al. (2010) used an additional characteristic quantifying the asymmetry of profiles with reversal. We did not use this here because it is sensitive mostly to the values of the LOS velocities, which are not the main interest of this study. The synthetic spectra obtained by the multi-thread model were resampled from the virtual slit scale (the actual $x-y$ grid of the model seen with an angle $\mu$ ) into the SUMER slit scale. One pixel along the SUMER slit is equivalent to one arcsec, which is equal to $726 \mathrm{~km}$ on the solar limb at the date of observations. The observed profiles of the Lyman lines are mainly blended by Balmer lines of He II and by UV lines of OI. These blends need to be taken into account when deriving the three statistical characteristics. Approximate contributions of blends to the Lyman-line profiles

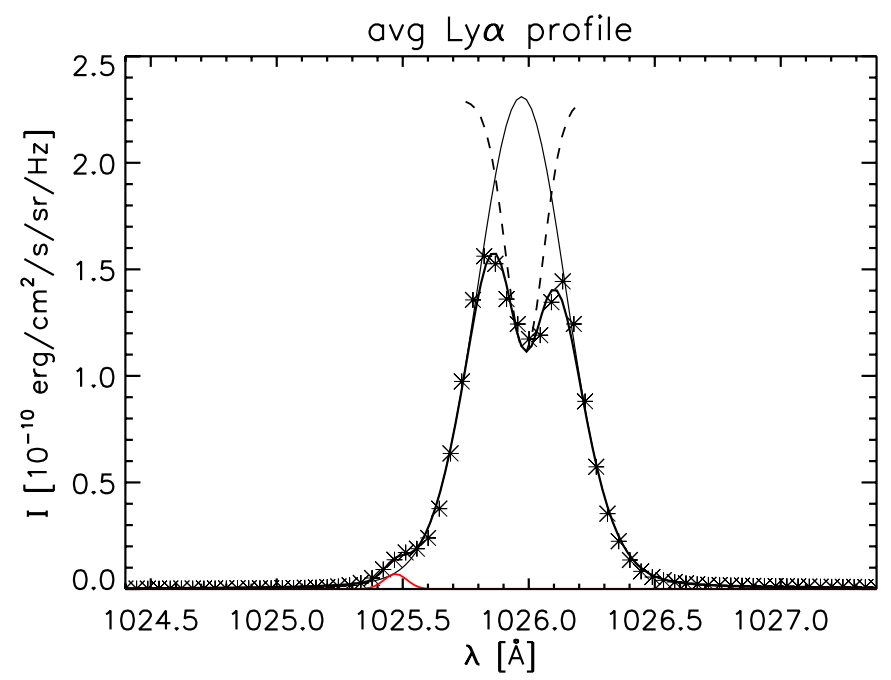

Fig. 5. Example of fitting of a $\operatorname{Ly} \alpha$ profile to estimate the influence of a blend on the three profile characteristics. Observed data are plotted with stars, while the resulting fitted profile (including blends) is plotted as a thick full line. The Voigt function fitting the profile in the wings is plotted as a thin solid line and the negative Gaussian in the profile reversal as a dashed line. The blend occurring at approximately $1025.5 \AA$ was fitted with the Gaussian and is plotted in red.

were estimated by fitting the blending lines with Gaussian functions, using the data averaged along the slit. The shape of the observed Lyman-line profiles were reproduced with the Voigt function in the wings and with a negative Gaussian in the central reversal, as is shown in an example of the Ly $\alpha$ profile in Fig. 5. This shows that even non-symmetrical profiles can be fitted by this method. The profiles were fitted with the LevembergMarquardt method. The negative Gaussian used for fitting the central reversals has no physical meaning, it was only used to reproduce the shape of the Lyman-line profiles without blends with the highest achievable accuracy. It is important to note that the fitting of the observed profiles was not used to derive the three profile characteristics. It was used only to estimate the influence of blends on the three profile characteristics estimated from the observed profiles. However, only blends located outside the Lyman-line cores that were sufficiently intense could be removed. For the $\operatorname{Ly} \alpha$ line the influence of blends on all profile characteristics is negligible. We estimate the contributions of blends to the integrated intensities of higher Lyman lines to be $2.8 \%$ for $\operatorname{Ly} \beta, 6.3 \%$ for $\operatorname{Ly} \gamma$, and $7.8 \%$ for $\operatorname{Ly} \delta$. These contributions were subtracted from the integrated intensities of the observed profiles. Blends do not significantly influence the other two statistical characteristics used here.

\section{Results}

\subsection{Multi-thread model with identical threads}

To obtain the best possible agreement between synthetic profiles obtained by the multi-thread model and the observations, we proceeded as follows. Several sets of single-thread models that each produced synthetic Ly $\alpha$ profiles similar to the observed ones were identified by trial and error. Then we selected from each such set the single-thread model with the least $\chi^{2}$ between the observed and synthetic Ly $\alpha$ profiles. Next, synthetic Ly $\alpha$ to Ly $\delta$ profiles were calculated for multiple configurations (random shifts and LOS velocities) of the multi-thread model with 

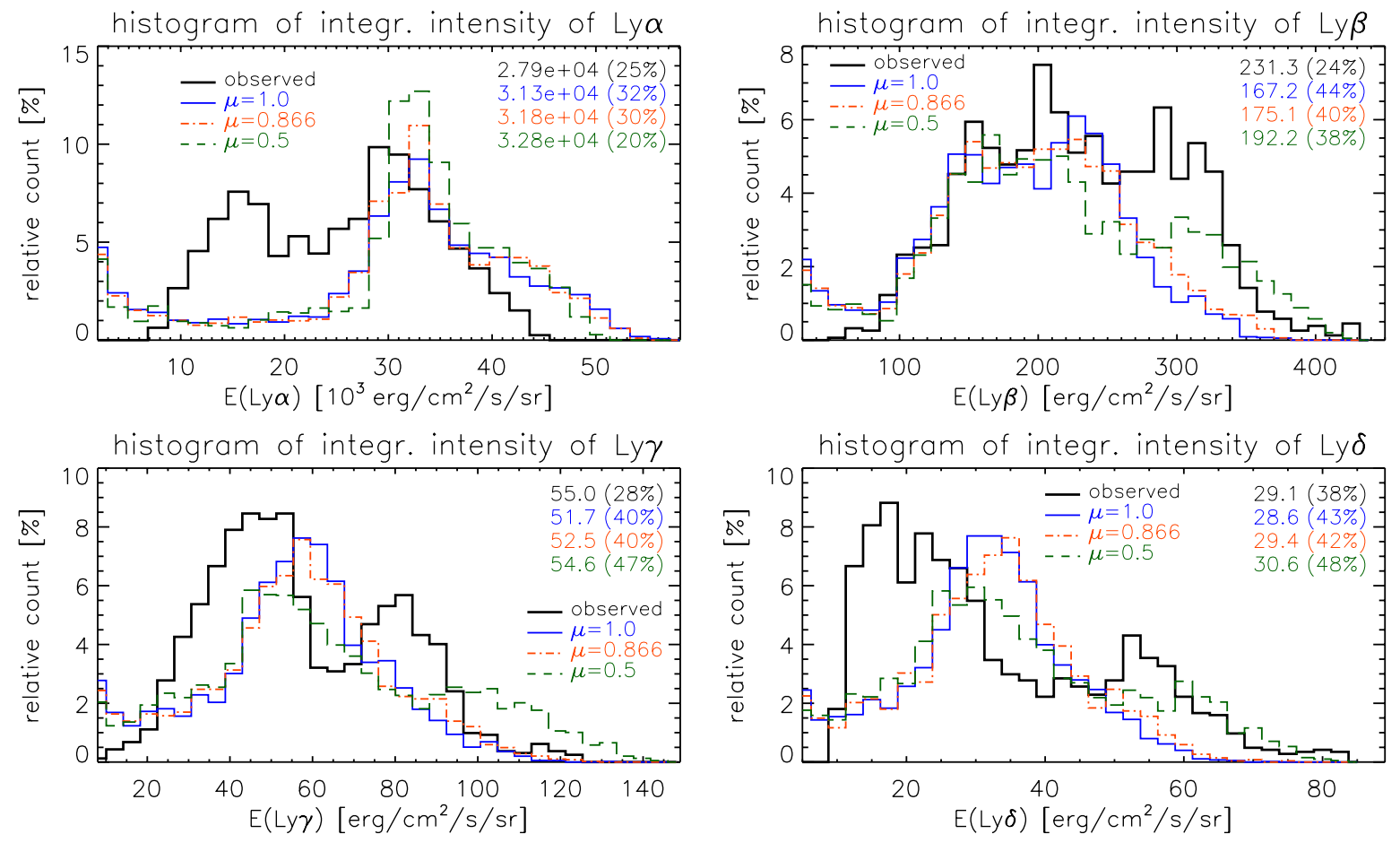

Fig. 6. Compared histograms of the integrated intensities of the observed and synthetic Ly $\alpha$ to $\operatorname{Ly} \delta$ line profiles. The solid black line represents the observed data. Three values of $\mu$ are represented by corresponding colours and line-styles.

ten identical threads for each of the selected single-thread models. We calculated the synthetic profiles for various LOS angles $\mu$. Three statistical characteristics of the synthetic profiles (see Sect. 3.3) were then compared with observations. Finally, a unique single-thread model with the best agreement of statistical characteristics of synthetic and observed profiles was identified. The input parameters of this model are the following:

$$
\begin{aligned}
& M_{0}=8.5 \times 10^{-5} \mathrm{~g} / \mathrm{cm}^{2}, T_{0}=8000 \mathrm{~K}, \quad p_{0}=0.02 \mathrm{dyn} / \mathrm{cm}^{2} \\
& \gamma_{1}=10, \quad \gamma_{2}=60, \quad B_{x}(0)=7 \mathrm{G} .
\end{aligned}
$$

The distributions of the temperature and gas pressure of this single-thread model are shown in Fig. 3.

The set of observed profiles contains 2160 Ly $\alpha$ profiles and 4320 profiles of each of the higher three Lyman lines. We compared the profile characteristics of such statistically significant set of observed data with the characteristics of 30000 synthetic profiles of each of the four Lyman lines calculated with the multi-thread model. That means 300 profiles along the virtual SUMER slit corresponding to the size of the multi-thread structure in 100 realisations of the multi-thread model.

In Fig. 6 we show the histograms of the integrated intensity of the $\operatorname{Ly} \alpha$ to $\operatorname{Ly} \delta$ synthetic profiles obtained with the multithread model with ten identical threads. For each thread the single-thread model with shown above input parameters was used. The synthetic profiles were calculated for three LOS directions - perpendicular to the magnetic field $(\mu=1)$ and at angles $30^{\circ}(\mu=0.866)$ and $60^{\circ}(\mu=0.5)$ from the direction perpendicular to the magnetic field. Each angle is represented by a specific colour and line-style. The solid black line represents the observed spectra. Numbers in the upper right corner in each panel of Fig. 6 are median values of histograms in corresponding colours. In parenthesis, a percentage value of the median of absolute deviations from the median is given. This provides information on the histogram dispersion. The histograms of the integrated intensities of the synthetic Ly $\alpha$ profiles only agree well with the observed data in their higher intensity peak (around $30000 \mathrm{erg} / \mathrm{cm}^{2} / \mathrm{s} / \mathrm{sr}$ ). The lower intensity peak at approximately $15000 \mathrm{erg} / \mathrm{cm}^{2} / \mathrm{s} / \mathrm{sr}$ is absent from the synthetic data. For $\operatorname{Ly} \beta$, the histograms of the synthetic integrated intensities have only one peak for $\mu$ equal to 1 and 0.866 , while the observed data show two peaks. Only the synthetic data histogram for $\mu=0.5$ has two peaks, and their positions agree with the observed data. For $\operatorname{Ly} \gamma$ and $\operatorname{Ly} \delta$, the situation is similar as for $\operatorname{Ly} \beta$, but the agreement between the positions of the two peaks in the histograms of the synthetic data for $\mu=0.5$ and observed data is poorer.

For the integrated intensity ratios of $\operatorname{Ly} \gamma / \operatorname{Ly} \beta$ and $\operatorname{Ly} \delta / \operatorname{Ly} \beta$ (Lyman decrement), the synthetic and observed data agree well for all three studied values of $\mu$, as can be seen in Fig. 7 . However, they agree less well for the $\operatorname{Ly} \alpha / \operatorname{Ly} \beta$ ratio. Although a narrow peak in the synthetic data histograms almost coincides with the observed data, to the right of it, a wide bulge appears that is absent from the observed data. This could be explained by the fact that the observed data only represent a small part of the whole prominence, while the synthetic profiles are produced along the whole length of the fine-structure threads.

The histograms of the centre-to-peak ratio of the synthetic $\operatorname{Ly} \alpha, \operatorname{Ly} \gamma$, and $\operatorname{Ly} \delta$ lines shown in Fig. 8 agree reasonably well with the observed data. Only the histogram for the $\operatorname{Ly} \beta$ line differs significantly - the maximum of the centre-to-peak ratio of the observed data is lower than the maximum of the synthetic data histograms. This difference contradicts the findings of Gunár et al. (2010), who found the synthetic $\operatorname{Ly} \beta$ profiles to have a much deeper central reversal than the observed profiles. From this brief analysis we conclude that the agreement between the observed and synthetic $\operatorname{Ly} \alpha$ to $\operatorname{Ly} \delta$ line profiles for $\mu$ between 0.5 and 0.866 (angles $30^{\circ}$ and $60^{\circ}$ ) is satisfactory. However, it is difficult to reproduce the observed data histograms of some profile characteristics with double-peak distributions using the multi-thread model with identical threads. 



Fig. 7. Compared histograms of the Lyman decrement of observed and synthetic Lyman-line profiles. The solid black line represents the observed data. Three values of $\mu$ are represented by corresponding colours and line-styles.

These double-peak distributions suggest threads with various plasma conditions. In the following subsection we assume for the first time multi-thread models with non-identical threads.

\subsection{Hotter threads at the edge of the prominence}

In this subsection we investigate whether hotter threads at the edge of the multi-thread model can improve the agreement between the synthetic and observed data. For the inner threads of this multi-thread model we used the same single-thread model as for identical threads. For the outer threads we assumed higher values of the minimum central temperature $T_{0}$, while other input parameters were left unchanged. For very high values of $T_{0}$ equal to $30000 \mathrm{~K}$ in the foremost and farthest threads and $15000 \mathrm{~K}$ in the second and penultimate threads (the 9th in our case), the histograms of integrated intensities of the synthetic $\operatorname{Ly} \alpha$ to $\operatorname{Ly} \delta$ profiles are much wider (4.8 times wider for Ly $\alpha$, 6.3 times for $\operatorname{Ly} \beta, 6.2$ times for $\operatorname{Ly} \gamma$, and 7 times for $\operatorname{Ly} \delta$ ) than for the observed data. The example for the Ly $\alpha$ line is shown in the left panel of Fig. 9. When lower $T_{0}$ values of $15000 \mathrm{~K}$ and $10000 \mathrm{~K}$ were used for the outer threads, the synthetic histograms of the integrated intensities are still approximately twice as wide as the observed ones - see example for Ly $\alpha$ in the right panel of Fig. 9. These results clearly demonstrate that any significant change of the input parameters even in a few threads of the multi-thread model produces large differences.

However, if values of $T_{0}$ in the outer threads are only slightly higher than in the inner threads, non-negligible changes occur only in a few histograms of the profile characteristics compared with histograms for multi-thread models with identical threads. We compare a model with marginally hotter outer threads (10000 and $9000 \mathrm{~K})$ with the identical-thread model and with observations in Fig. 10. We show only the histograms with a non-negligible differences from the identical-thread model. The histograms for those values of $\mu$ are shown here that agree best with observations. The histograms of the integrated intensity of the synthetic $\operatorname{Ly} \alpha$ and $\operatorname{Ly} \beta$ lines agree moderately worse with observations for models with outer threads with $T_{0}$ of 10000 and $9000 \mathrm{~K}$ than for identical threads. In contrast, using outer threads with $T_{0}$ of 10000 and $9000 \mathrm{~K}$ improves agreement of the histograms of the integrated intensity of the synthetic Ly $\gamma$ and $\operatorname{Ly} \delta$ line-profiles with observations. The histograms of the Lyman decrement are almost the same as those for the models with identical threads and are therefore not shown in Fig. 10.

The histograms of the centre-to-peak ratio of the synthetic Ly $\alpha$ line profiles obtained using the models with marginally hotter outer threads reproduce the observed histogram much better than the models with identical threads (see Fig. 10). For Ly $\beta$, neither the model with outer threads with $T_{0}$ of 10000 and $9000 \mathrm{~K}$ nor identical-thread model reproduce the observed histogram. Only much higher values of $T_{0}$ in the outer threads (such as $T_{0}$ of 30000 and $15000 \mathrm{~K}$ ) would produce histograms of the synthetic Ly $\beta$ centre-to-peaks ratio that are comparable with the observations. However, the histograms of integrated intensities are much wider than the observed ones at the same time (see Fig. 9).

\section{Discussion}

The multi-thread model with identical threads is not able to reproduce the histogram of the observed Ly $\alpha$ integrated intensity with two peaks. The reason for this might be that in reality threads in prominences are probably not identical. To investigate this idea, we used for the first time 2D multi-thread models with non-identical threads. We placed two hotter threads on either sides of the multi-thread model while keeping the total number of threads equal to 10 . Assuming central temperatures $T_{0}$ above $10000 \mathrm{~K}$ in the outer threads while keeping inner threads temperature $T_{0}$ at $8000 \mathrm{~K}$ leads to histograms of integrated intensities of the synthetic $\operatorname{Ly} \alpha$ to $\operatorname{Ly} \delta$ lines that are much wider than the observed data histograms. When using central temperatures $T_{0}$ of the outer threads that are only one and two thousand $\mathrm{K}$ higher than in the inner threads, the histograms of the centre-to-peak ratio of the synthetic Ly $\alpha$ line with single peak were obtained for all three LOS angles $0^{\circ}, 30^{\circ}$ and $60^{\circ}$ (corresponding to $\mu$ values of $1,0.866$, and 0.5 ). This agrees well with the observed Ly $\alpha$ line histogram that likewise has a single peak. In contrast, the multi-thread model with identical threads produces histograms with two-peak distribution of the Ly $\alpha$ centreto-peak ratio. The histograms of the centre-to-peak ratio for the synthetic $\operatorname{Ly} \beta, \operatorname{Ly} \gamma$ and $\operatorname{Ly} \delta$ lines differ only slightly from those obtained with the identical-thread model. However, using the hotter outer threads does not lead to histograms of integrated intensity of the synthetic Ly $\alpha$ profiles with two peaks as in the observed data. We also ran tests with outer threads having a lower central column mass $M_{0}$. However, these models did not 

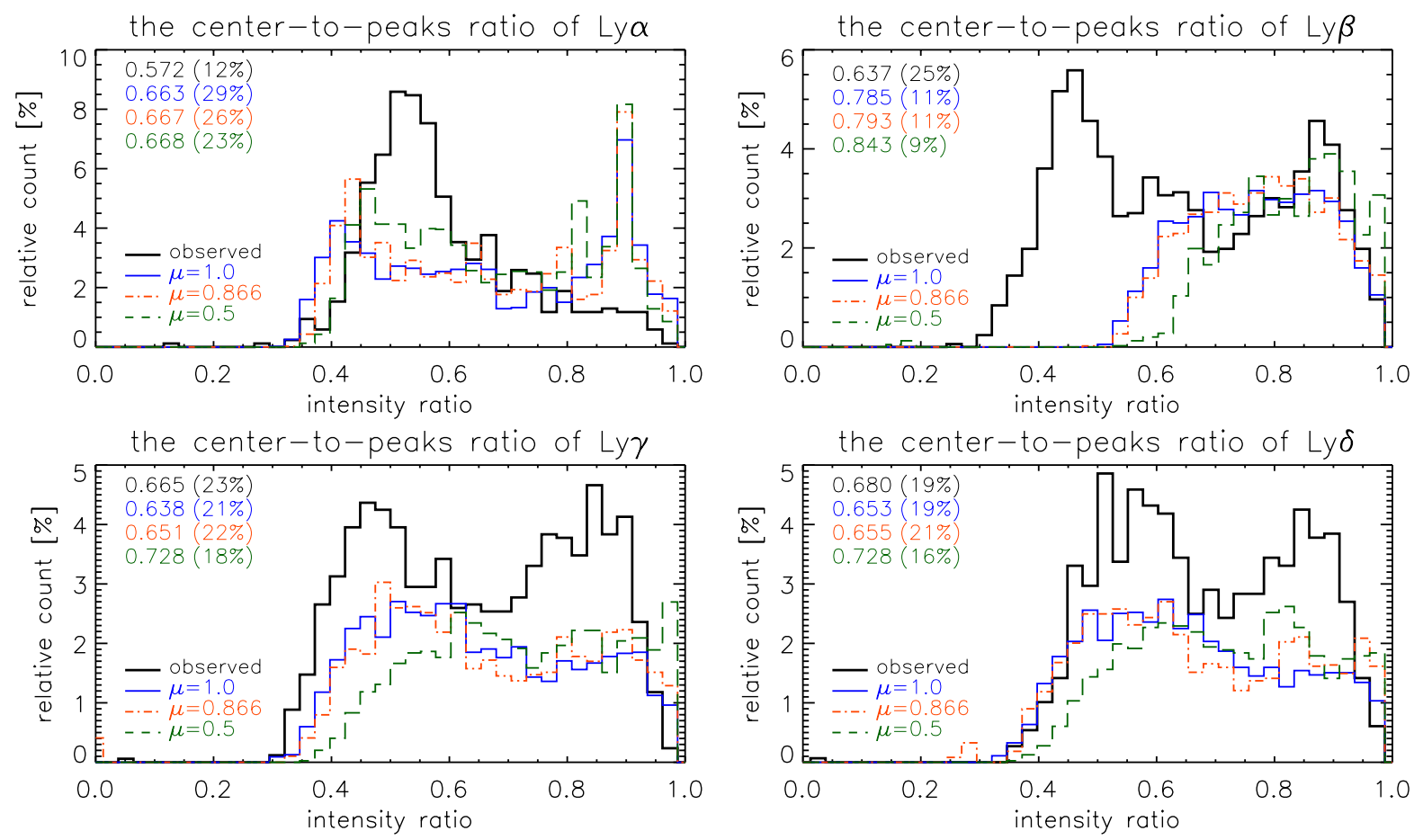

Fig. 8. Histograms of ratio of the intensity at the central reversal to mean intensity from peaks for the observed and synthetic profiles of the Ly $\alpha$ to Ly $\delta$ lines. The solid black line represents the observed data. Three values of $\mu$ are represented by corresponding colours and line-styles.

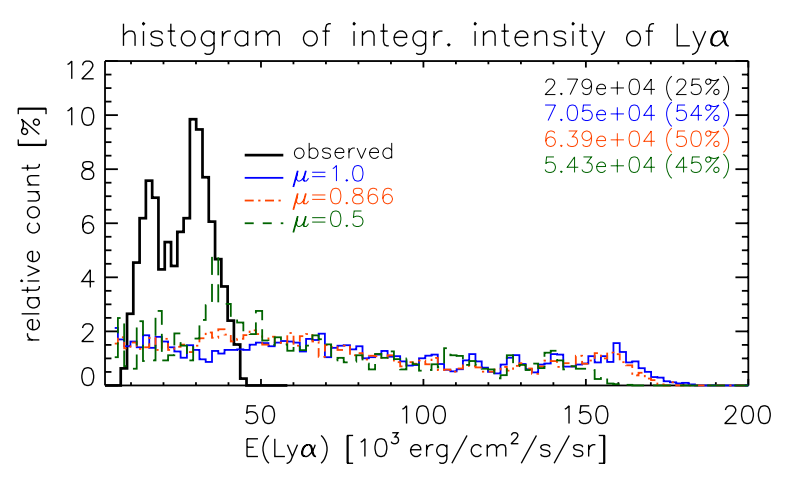

$T_{0}$ in outer threads: 30000 and $15000 \mathrm{~K}$

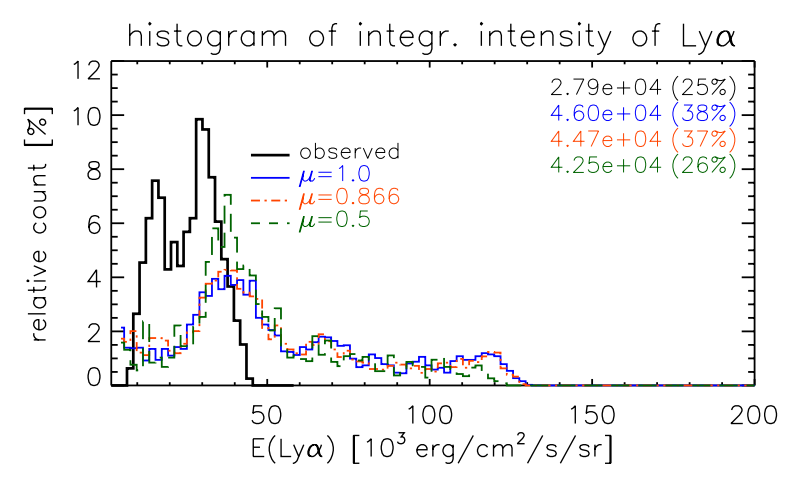

$T_{0}$ in outer threads: 15000 and $10000 \mathrm{~K}$

Fig. 9. Compared histograms of the $\operatorname{Ly} \alpha$ integrated intensity of the observed profiles with those calculated using multi-thread models with hotter outer threads. Left panel: multi-thread models with $T_{0}$ values of 30000 and $15000 \mathrm{~K}$ in the outer threads. Right panel: comparison for somewhat lower temperatures of 15000 and $10000 \mathrm{~K}$. The solid black line represents the observed data. Three values of $\mu$ are represented with corresponding colours and line-styles.

lead to a double-peaked distributions of the synthetic Ly $\alpha$ integrated intensities. For example, using $M_{0}$ lower by an order of magnitude, $1 \times 10^{-5} \mathrm{~g} \mathrm{~cm}^{2}$, in the outer threads (MoDEL_2 of Berlicki et al. 2011) only shifts the histograms of the integrated intensities and the centre-to-peak ratio to lower values. It can be also argued that the temporal changes of the prominence structure occurring during the observations cannot be the cause of the double-peak distribution of the observed Ly $\alpha$ integrated intensities. This is because variations of the Ly $\alpha$ integrated intensities between individual spectra obtained at different times are significantly smaller (lower than $40 \%$ ) than the difference between the peaks in the histogram. The lower intensity peak in the histogram could be caused by the apparent spatial separation of the lower and higher intensity Ly $\alpha$ profiles within the spectra. The lower-intensity profiles tend to cluster in the southern part of the slit, which is probably located outside of the bright part of the observed prominence (see Fig. 1, left). However, without cotemporal high-resolution $\mathrm{H} \alpha$ observations we cannot study this spatial distribution in detail. The disagreement for histograms of the $\operatorname{Ly} \alpha$ and $\operatorname{Ly} \beta$ centre-to-peak ratio might be explained by neglecting the mutual radiative interaction between individual threads. As was shown by Heinzel et al. (2010) using 2D multithread models with three identical threads, the mutual radiative interaction mainly influences peaks of the Lyman-line profiles, thus mainly histograms of the centre-to-peak ratio are affected. Heinzel et al. (2010) found that the most intense profiles have the highest increase of intensities at peaks and that the influence of the mutual radiative interaction decreases with the distance between threads. Thus, with increasing intensities at peaks, the number of deeper profiles increases, while the number of shallower profiles at the same time decreases. This shifts the maxima of the centre-to-peak ratio histograms towards lower values. The 
P. Schwartz et al.: Modelling of prominence fine structure
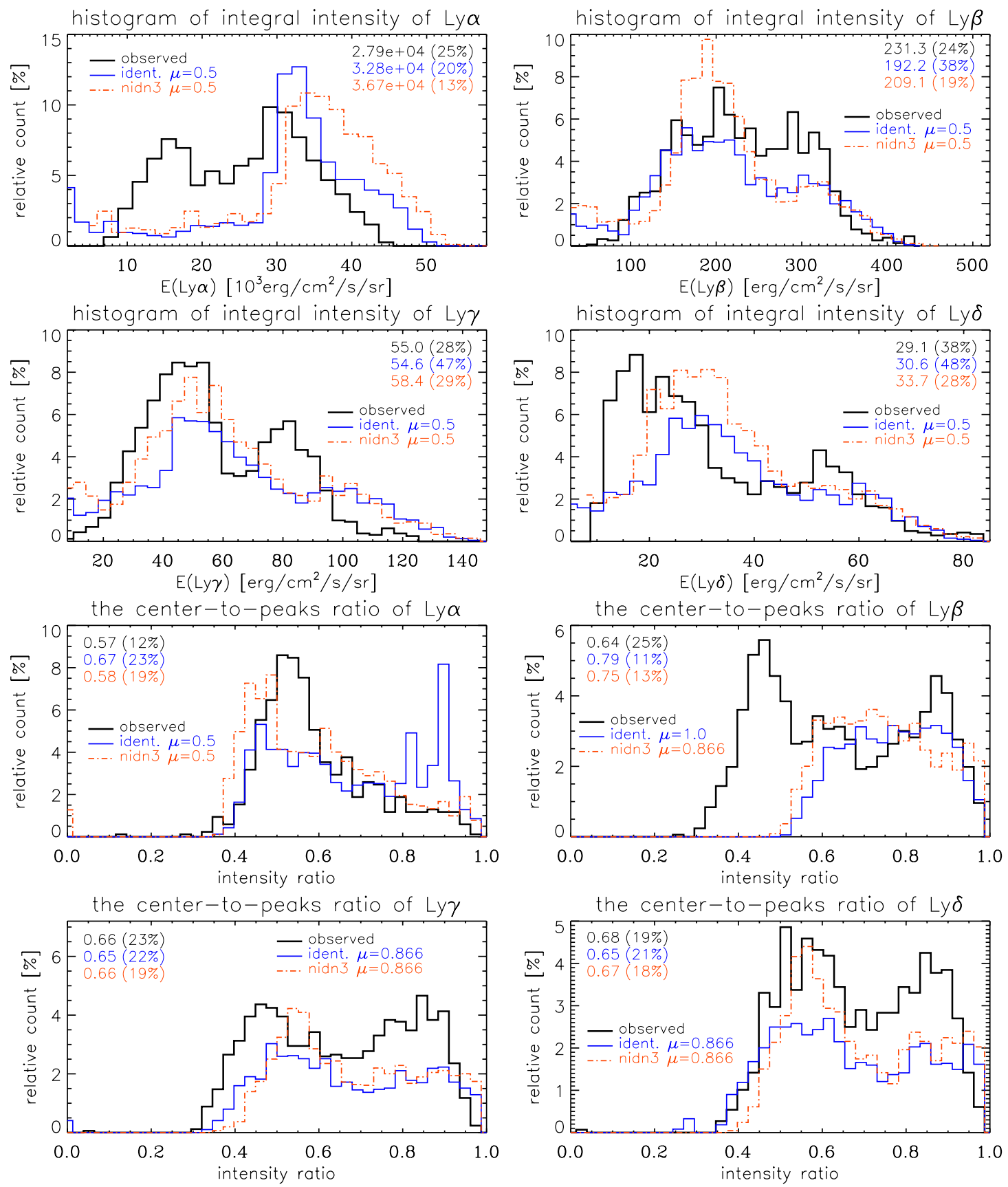

Fig. 10. Compared histograms of selected Lyman-line profile characteristics for synthetic profiles calculated using the models with identical threads (denoted as ident.) and models with outer threads with temperatures $T_{0}$ of 9000 and $10000 \mathrm{~K}$ (denoted as nidn) with histograms of characteristics of observed profiles (solid black line).

effect of the mutual radiative interaction is strongest mainly for the $\operatorname{Ly} \alpha$ and $\operatorname{Ly} \beta$ lines. In contrast, Heinzel (1989) found an opposite effect for a $1 \mathrm{D}$ multi-thread model with 40 threads - the intensity at peaks decreased when mutual interaction was taken into account. This discrepancy between the findings of Heinzel (1989) in 1D and of Heinzel et al. (2010) in 2D geometry clearly shows that the question of the effect of the mutual radiative interaction is still unanswered. We plan to study it in detail in future with 2D multi-thread models.

\section{Conclusions}

We analysed the quiescent prominence observed on NW limb on May 18, 2005 in detail. Using profile-to-profile comparison of the observed $\operatorname{Ly} \alpha$ profiles with the synthetic Ly $\alpha$ profiles obtained by the single-thread model, we found a set of input parameters representing a rather dense prominence with the central total column mass $M_{0} \sim 10^{-4} \mathrm{~g} / \mathrm{cm}^{2}$. This is similar to MODEL1 found by Gunár et al. (2010). For the statistical analysis we used three characteristics of the Lyman-line profiles: the integrated intensity, the Lyman decrement ratio, and the ratio of intensity at the central reversal to average intensity of peaks. The multi-thread model with ten identical threads used for this statistical analysis agrees satisfactorily with observations for histograms of the integrated intensities of synthetic and observed $\operatorname{Ly} \alpha$ to $\operatorname{Ly} \delta$ line profiles for angles $30^{\circ}$ and $60^{\circ}$ (corresponding to the $\mu$ of 0.866 and 0.5 ) between the LOS and the direction perpendicular to the magnetic field. LOS angles different from 
perpendicular were used here for the first time. However, the multi-thread model with identical threads is not capable to reliably reproduce the two-peaked histograms of the observed Ly $\alpha$ integrated intensities. In addition, while the histograms of the Lyman decrements of $\operatorname{Ly} \gamma / \operatorname{Ly} \beta$ and $\operatorname{Ly} \delta / \operatorname{Ly} \beta$ agree very well with observations, the $\operatorname{Ly} \alpha / \operatorname{Ly} \beta$ histograms of the synthetic data are considerably wider (due to the double-peak distribution of the Ly $\alpha$ integrated intensities) than the histograms of the observed data. Histograms of the centre-to-peak ratio for the synthetic Ly $\alpha$ and $\operatorname{Ly} \beta$ lines fit the observed data only partially, while for Ly $\gamma$ and $\operatorname{Ly} \delta$ the agreement is satisfactory. Gunár et al. (2010) found for a different prominence also agreement of the centre-to-peak histograms with observations only for $\operatorname{Ly} \gamma$ and $\operatorname{Ly} \delta$. But the discrepancy between their Ly $\alpha$ and $\operatorname{Ly} \beta$ synthetic and observed data is much more significant.

To investigate the idea that the double-peak histograms of the observed Ly $\alpha$ integrated intensities might be caused by threads with various plasma parameters, we used here for the first time multi-thread models with non-identical threads. When we used relatively hot threads $\left(T_{0}>10000 \mathrm{~K}\right)$ at the edges of the multithread model, the synthetic spectra histograms of integral intensities were significantly different from the observed data histograms. This clearly shows that a relatively large variation of parameters of multi-thread model, even in a few of the threads, produces a very large difference in the synthetic spectra. On the other hand, when we used outer threads with slightly higher temperatures $\left(T_{0}=10000\right.$ and $\left.9000 \mathrm{~K}\right)$, only some of the statistical parameters were significantly affected, improving the agreement with observations in several cases and deteriorating it in others. Therefore we argue that the temperature in the interiors of cool prominence fine structures in the studied prominence is at about $8000 \mathrm{~K}$ and does not vary significantly among individual structures. This means that the multi-thread model with identical threads is sufficient for a statistical comparison of observed and synthetic data. However, none of the multi-thread models with non-identical threads was able to reproduce the double-peak histogram of the observed Ly $\alpha$ integrated intensities.

The results of the multi-thread model with hotter threads at its edges does not suggest that areas with plasma of higher temperatures do not exist in prominences. The visibility of prominences in the transition-region (TR) lines was previously demonstrated by Poland \& Tandberg-Hanssen (1983) on UV observations of a prominence obtained on November 20, 1980 by the Ultraviolet Spectrometer and Polarimeter (Woodgate et al. 1980) onboard the Solar Maximum Mission. For filaments, the TR O v line emission from PCTR was assumed in Schwartz et al. (2004). However, results presented here show that extended regions of prominences filled with plasma at the lower limit of PCTR temperatures (below $30000 \mathrm{~K}$ ) would have a significant effect on the Lyman spectra emitted by prominences.
Acknowledgements. P.S. acknowledges support from grant P209/12/0906 of the Grant Agency of the Czech Republic. S.G. acknowledges support from the European Commission via the Marie Curie Actions - Intra-European Fellowships Project No. 328138. The work of P.S. was supported by project RVO: 67985815. P.S. acknowledges support from the Slovak Research and Development Agency under the contract No. APVV-0816-11. The work of P.S. was supported by grant project VEGA 2/0108/12 of the Science Grant Agency. S.G. thanks for support by the Astronomical Institute of the Academy of Sciences of the Czech Republic. We are thankful to P. Heinzel for providing us with his 2D non-LTE code for solving radiative transfer in solar prominences. We are also thankful to the anonymous referee for useful comments and suggestions.

\section{References}

Anzer, U., \& Heinzel, P. 1998, Sol. Phys., 179, 75

Anzer, U., \& Heinzel, P. 1999, A\&A, 349, 974

Auer, L. H., \& Paletou, F. 1994, A\&A, 285, 675

Berlicki, A., Gunár, S., Heinzel, P., Schmieder, B., \& Schwartz, P. 2011, A\&A, 530, A143

Curdt, W., Brekke, P., Feldman, U., et al. 2001, A\&A, 375, 591

Goode, P. R., Denker, C. J., Didkovsky, L. I., Kuhn, J. R., \& Wang, H. 2003, J. Kor. Astron. Soc., 36, 125

Gouttebroze, P. 2006, A\&A, 448, 367

Gouttebroze, P., Heinzel, P., \& Vial, J. C. 1993, A\&AS, 99, 513

Gunár, S. 2014, in IAU Symp., 300, 59

Gunár, S., Heinzel, P., Schmieder, B., Schwartz, P., \& Anzer, U. 2007, A\&A, 472,929

Gunár, S., Heinzel, P., Anzer, U., \& Schmieder, B. 2008, A\&A, 490, 307

Gunár, S., Schwartz, P., Schmieder, B., Heinzel, P., \& Anzer, U. 2010, A\&A 514, A43

Gunár, S., Heinzel, P., \& Anzer, U. 2011a, A\&A, 528, A47

Gunár, S., Parenti, S., Anzer, U., Heinzel, P., \& Vial, J.-C. 2011b, A\&A, 535 A122

Gunár, S., Mein, P., Schmieder, B., Heinzel, P., \& Mein, N. 2012, A\&A, 543, A93

Gunár, S., Schwartz, P., Dudík, J., et al. 2014, A\&A, 567, A123

Heasley, J. N., \& Mihalas, D. 1976, ApJ, 205, 273

Heinzel, P. 1989, Hvar Observatory Bulletin, 13, 317

Heinzel, P. 2007, in The Physics of Chromospheric Plasmas, eds. P. Heinzel, I. Dorotovič, \& R. J. Rutten, ASP Conf. Ser., 368, 27

Heinzel, P., \& Anzer, U. 2001, A\&A, 375, 1082

Heinzel, P., Anzer, U., \& Gunár, S. 2010, Mem. Soc. Astron. It., 81, 654

Kunasz, P., \& Auer, L. H. 1988, J. Quant. Spectr. Rad. Transf., 39, 67

Labrosse, N., Heinzel, P., Vial, J., et al. 2010, Space Sci. Rev., 151, 243

Mackay, D. H., Karpen, J. T., Ballester, J. L., Schmieder, B., \& Aulanier, G. 2010, Space Sci. Rev., 151, 333

Mihalas, D., Auer, L. H., \& Mihalas, B. R. 1978, ApJ, 220, 1001

Patsourakos, S., \& Vial, J.-C. 2002, Sol. Phys., 208, 253

Poland, A., \& Anzer, U. 1971, Sol. Phys., 19, 401

Poland, A. I., \& Tandberg-Hanssen, E. 1983, Sol. Phys., 84, 63

Schühle, U. 2003, SUMER Data Cookbook, Published at http://www.mps. mpg.de/projects/soho/sumer/text/cookbook.html

Schwartz, P., Heinzel, P., Anzer, U., \& Schmieder, B. 2004, A\&A, 421, 323

Schwartz, P., Schmieder, B., Heinzel, P., \& Kotrč, P. 2012, Sol. Phys., 281, 707

Wilhelm, K., Curdt, W., Marsch, E., et al. 1995, Sol. Phys., 162, 189

Woodgate, B. E., Brandt, J. C., Kalet, M. W., et al. 1980, Sol. Phys., 65, 73 